\title{
A Psychotherapy Service
}

\section{How general practitioners see it}

StePhen P. Rellly, Consultant Psychiatrist with Special Responsibility for Psychotherapy, Bootham Park Hospital, Bootham, York (formerly Senior Registrar in Psychotherapy, Manchester Royal Infirmary)

Over recent years Central Manchester GPs have supplied over $50 \%$ of the referrals to the Gaskell House Department of Psychotherapy. Yet there is no formal channel for feedback from these GPs concerning the services provided and for opportunites for realistic enhancement of the relationship between them and the predominantly hospital-based psychotherapists. It would appear that this communication gap applies to many psychotherapy centres in the UK though Tyson and Reder ${ }^{1}$ have specified the opinions of GPs as one variable to be considered when planning psychotherapy service.

There are few relevant references. In Manchester it is not known how many patients are currently referred to psychiatric clinics with a specific request for psychotherapy but Johnson ${ }^{2}$ found in an analysis of out-patient services that only $8 \%$ of all new referrals to three psychiatric units in the city were treated with psychotherapy alone. In another Manchester study of the treatment of depression in general practice Johnson ${ }^{3}$ found that only 2 out of 73 depressed patients were offered psychotherapy and that the doctors had a limited knowledge of its scope and techniques. Eleven years previously, in 1962, a Welsh study ${ }^{4}$ had found that GPs did not offer psychotherapy as they doubted their ability to provide it effectively.

In contrast with the provinces the wide availability of psychotherapy services in London means that the GP has little difficulty finding psychotherapeutic help for his patients. In one study Kalena ${ }^{5}$ discovered that $41 \%$ of all psychiatric patients from a particular practice were referred to out-patients with a specific request for individual analytic psychotherapy'.

In Manchester there is a growing demand for psychotherapy, particularly for the more seriously disturbed patient and, given the new Griffiths' climate of 'efficiency', 'value for money' and intense interdisciplinary competition for resources it is vitally important that NHS psychotherapy facilities are seen to make a contribution to health care.

Enlisting the views and support of referring agencies is one aspect of this complex issue. The pilot study described below (conducted in 1984-85) aimed to assess the feasibility of obtaining relevant information from GPs concerning their utilisation of psychotherapy services, knowledge of the range of therapies available, and views on the usefulness or otherwise of the services on offer.

The rationale for carrying out this study applies to other psychotherapy centres in the UK and, although the results are of limited local application only they may be of interest to stimulate investigations elsewhere.

\section{Background to the study}

Central Manchester psychotherapy services are based at Gaskell House, Manchester Royal Informary. A comprehensive range of treatments are available including individual, group, marital and family therapies. Psychodynamic, behavioural and cognitive approaches are all represented. The two consultant psychotherapists and associated team members operate both district and regional services with inpatient, and out-patient facilities. The clinical psychologists operate a district out-patient service within both hospital and primary care settings. There are $\mathbf{4 1}$ general practices sited within the approximate geographical area of Central Manchester and they vary in size from one to ten doctors. GPs from the University of Manchester Student Health Service were excluded from the study because they work outside the NHS and anyway, have a special liaison link with Gaskell House.

The catchment area includes inner city and suburban wards with a total population of approximately 131,000 .

\section{Method}

All 108 GPs listed by the Family Practitioner Committee as working in Central Manchester were asked to complete a postal questionnaire which covered: (i) qualifications and experience; (ii) utilisation of psychotherapy services; (iii) knowledge of the range of therapies available and (iv) views on the usefulness of the services. The results are categorised below. Figures are given to the nearest whole number.

\section{Results}

Response: The practice response rate was $85 \%$ (35 practices); the individual rate, $68 \%$ (73 GPs). The group consisted of 10 women and 63 men. Most GPs had obtained some or all of their qualifications in the UK $(84 \%)$. The duration of clinical experience ranged from 1-45 years, with a mean of 20 years.

A general interest in the psychotherapies was declared by $81 \%$ of doctors and $85 \%$ knew of the availability of psychotherapy services at Gaskell House. Support for the services as necessary and worthwhile was forthcoming from $95 \%$ of respondents.

GPs were split on the issue of whether to refer potential psychotherapy cases to a psychiatrist for initial assessment $(51 \%)$ or directly to a psychotherapist $(40 \%)$. 
Over the preceding 12 months $34 \%$ of GPs had referred patients to the psychotherapy unit. There was no statistically significant correlation between a declared interest in psychotherapy and a tendency to refer patients $\left(X^{2}=1.30\right)$.

Most referrals are seen within six weeks of completion and return of a questionnaire which is filled in by the patient. A total of $79 \%$ of GPs were satisfied that referrals were seen quickly enough and $92 \%$ agreed that sufficient information was fed back to them about assessment and treatment plans.

Awareness of the availability of particular psychotherapies varied greatly. Many GPs knew of the availability of individual $(79 \%)$ and group therapy $(70 \%)$ but only $26 \%$ knew of the family therapy clinic. Availability of behavioural approaches was known to $66 \%$ whilst only $26 \%$ were sure that dynamic psychotherapy was on offer. The same number $(26 \%)$ knew that cognitive therapy was available. Most GPs (92\%) wanted to know more about the psychotherapy services.

Requests for specific comments on the services brought a response from $27 \%$ of GPs, which fell into five categories: (i) requests for further education and training; (ii) requests for closer working contacts between general practitioner and psychotherapist; (iii) praise for the services; (iv) scepticism of the value of psychotherapy (one GP only); and (v) the need for psychotherapy facilities nearer patients' homes.

\section{Discussion}

Clearly it is feasible to obtain an outline of GP' views on local psychotherapy services in a systematic way. The questionnaire response rate was good, particularly in terms of practice response.

Most responders believe that psychotherapy services are necessary and refer patients when appropriate. Even if all $32 \%$ of doctors who did not reply view the services unfavourably there are still $64 \%$ in favour.

Kalena's statement that "the degree to which the GP has a personal interest in psychodynamic matters will influence his utilisation of psychotherapy services" was not confirmed by the present study in which lack of interest in psychotherapy did not prevent referral.
Although the services were viewed positively by almost all GPs there were some valuable suggestions on possible improvement. Many GPs wanted more information about the services available; some wanted further training and supervision in order to manage more of their own patients. One GP expressed a strong interest in having a psychotherapist attached to his practice.

There requests accord well with the views of Cawley ${ }^{6}$ whose scheme requires that all health care professionals should achieve a level of competence in psychotherapy skills commensurate with the demands of their work. It is the responsibility of fully trained specialist psychotherapists (psychodynamic and behavioural) to train staff in their own fields and others to the required level.

Perhaps more time should be devoted to providing training, supervision and support to those doctors (and other disciplines) who want it. Also, more attention could be focused on telling GPs about the psychotherapy facilities available.

It is interesting that more than twice as many doctors knew of the availability of behaviour therapy as compared with dynamic psychotherapy. The likely explanation is that the clinical psychologists have some sessions in primary care settings and have more opportunity to educate GPs than the dynamically-oriented therapists who operate almost exclusively from a hospital base. The lesson is obvious.

\section{REFERENCES}

${ }^{1}$ Tyson, R. L. \& REDER, P. (1979) Questions of supply and demand in dynamic psychotherapy، British Journal of Medical Psychology, 52, 301-307.

${ }^{2} \mathrm{~J}$ JoHNSON, D. A. W. (1973) An analysis of out-patient service. British Journal of Psychiatry, 122, 301-306.

3 _ (1973) Treatment of depression in general practice. British Medical Journal, 2, 18-20.

${ }^{4}$ RAWNsLey, K. \& Loudon, J. B. (1962) Factors influencing the referral of patients to psychiatrists by general practitioners. British Journal of Preventative and Social Medicine, 16, 174-182.

SKalena, M. A. (1976) Psychiatric referral. Journal of the Balint Society, 5, 3-10.

${ }^{6}$ CAWLEY, R. H. (January 1977). The teaching of psychotherapy. Association of University Teachers in Psychiatry Newsletter. 19-36.

\section{Clinic for Treatment of Male Impotence}

The first clinic for the treatment of male impotence in Britain opened at the new Glen Hospital, Bristol, on 30 January. The clinic will offer patients a counselling and treatment service from urologists, psychologists and specially trained counsellors. Patients will be referred there by their GPs and other health care professionals for initial screening and diagnosis. Members of the public seeking advice will also be able to contact the clinic on a special direct telephone line (0272 744166). 